\title{
ON FACTORIZATIONS OF SELFADJOINT ORDNARY DIFFERENTIAL OPERATORS
}

\author{
ANTONIO GRANATA ${ }^{1}$
}

\begin{abstract}
Consider an ordinary linear differential operator $L$, of order $n \geqslant 1$, represented by $L u \equiv a_{n}(t) u^{(n)}+\cdots+a_{0}(t) u \quad \forall u \in C^{n}(a, b)$, with real-valued coefficients $a_{k} \in C^{k}(a, b), 0 \leqslant k \leqslant n, a_{n} \neq 0$ on $(a, b)$. According to a classical result, if $L$ is formally selfadjoint $\operatorname{lon}(a, b)$ then it has a factorization of the type $L u \equiv p_{n}\left[p_{n-1}\left(\cdots\left(p_{1}\left(p_{0} u\right)^{\prime}\right)^{\prime} \cdots\right)^{\prime}\right]^{\prime} \forall u \in C^{n}(a, b)$, where the $p_{k}$ 's are sufficiently-smooth and everywhere nonzero functions on $(a, b)$ such that $p_{k}=p_{n-k}$ $(k=0, \ldots, n)$. In this note we shall examine this result critically and show by means of counterexamples that the different classical proofs are either merely local or purely heuristic. A proof, which is both rigorous and global, is inferred from recent results on canonical factorizations of disconjugate operators. In addition, information is obtained on the behavior of the $p_{k}$ 's at the endpoints of $(a, b)$ which may prove useful in applications.
\end{abstract}

1. Introduction. Let $L$ be a linear ordinary differential operator represented by

$$
L u \equiv a_{n}(t) u^{(n)}+a_{n-1}(t) u^{(n-1)}+\cdots+a_{0}(t) u \quad \forall u \in C^{n}(a, b)
$$

with real-valued coefficients $a_{k} \in C^{0}(a, b), a_{n} \neq 0$ on $(a, b)$ and $n \geq 1$. It is a classical elementary fact that if $L$ has a factorization of the type

$$
L u \equiv p_{n}\left[p_{n-1}\left(\cdots\left(p_{1}\left(p_{0} u\right)^{\prime}\right)^{\prime} \cdots\right)^{\prime}\right]^{\prime} \quad \forall u \in C^{n}(a, b)
$$

where the $p_{k}$ 's are suitable sufficiently-smooth and everywhere nonzero functions on $(a, b)$, then its formal adjoint $L^{*}$ has the factorization

$$
L^{*} v \equiv(-1)^{n} p_{0}\left[p_{1}\left(\cdots\left(p_{n-1}\left(p_{n} v\right)^{\prime}\right)^{\prime} \cdots\right)^{\prime}\right]^{\prime} \quad \forall v \in C^{n}(a, b) .
$$

This is also a consequence of the classical reciprocity theorem (Reciprocitätsatz) by Frobenius $[3$, p. $263 ; 5$, p. 328; 6, p. 189] which states that the formal adjoint of the composition product of two differential operators, $P=A_{1} A_{2}$, is $P^{*}=A_{2}^{*} A_{1}^{*}$. Direct proofs of the relationship between (1.2) and (1.3) can also be found in Frobenius [4, p. 257] or Coppel [1, p. 104].

Now let a factorization of type (1.2) be called selfadjoint on $(a, b)$ when

$$
p_{k}(t)=\alpha_{k} p_{n-k}(t), \quad t \in(a, b), k=0,1, \ldots, n,
$$

for some nonzero constants $\alpha_{k}$. A direct application of the reciprocity theorem shows that if $L$ has a selfadjoint factorization on $(a, b)$ then it is formally selfadjoint on $(a, b)$; i.e., by definition, $L u=(-1)^{n} L^{*} u \forall u \in C^{n}(a, b)$. The converse has been long since given in the literature; we shall state it as

Received by the editors August 25, 1981.

1980 Mathematics Subject Classification. Primary 34A99; Secondary 34C10.

Key words and phrases. Selfadjoint, canonical factorizations.

${ }^{1}$ Paper written while the author was a member of the Italian Committee for Scientific Research C.N.R.-G.N.A.F.A. 
ASSERTION A. If $L$ is formally selfadjoint on an open interval $(a, b),-\infty \leq a<$ $b \leq+\infty$, then it admits of a selfadjoint factorization on $(a, b)$.

Assertion A goes back to Frobenius [6, p. 193] for $n$ even and to Darboux [2, livre IV, Chapitre V, p. 127] for $n$ odd. Their arguments are reported in the treatise by Schlesinger [13, Band I, Abschnitt II, Kapitel 3, pp. 70-75]. An apparently shorter proof appears in a book by Ince [10, pp. 125-126]. The Frobenius proof, though formally correct, does not take into due consideration the fact that several steps in obtaining factorizations of type (1.2) require multiplication by $1 / p_{k}$, hence they are only valid on an interval where $p_{k} \neq 0 \forall k$. Such a fact was alien to all the nineteenth century authors. It was not until 1922 that this point was first stated precisely by Polya [12] who characterized the existence of a factorization of type (1.2) with the property nowadays known as "disconjugacy". The original Frobenius proof is as follows: firstly [6, p. 192], he proves that the given operator $P$, assumed selfadjoint and of even order, can be written in the form $P u=c_{0} D P_{1} D\left(c_{0} u\right)$ with $c_{0}=c_{0}(t)$ such that $P\left(1 / c_{0}\right)=0$ (hence necessarily $c_{0} \neq 0$ ); then by applying the same procedure to the operator $P_{1}$ he infers that $P_{1}=c_{1} D P_{2} D\left(c_{1} u\right)$. When Polya's results are taken into consideration we can by no means be sure a priori that there exists a suitable function $c_{1} \neq 0$ on the same interval where $c_{0}$ is defined. Even if we assume at the outset that $P$ is disconjugate on $(a, b)$, and hence has a factorization of type (1.2), we cannot infer without further investigations the existence of such a $c_{1} \neq 0$ on $(a, b)$.

The arguments used by Darboux for odd-order operators are similar.

Taking account of Polya's results we may assert that the proofs by Frobenius and Darboux have a merely local value: these authors prove the existence of a selfadjoint factorization on every sufficiently small subinterval of $(a, b)$.

On the other hand, the proof given by Ince is indeed no proof: this author [10, p. 125], after showing that (1.2) implies (1.3), claims that if, in particular, $L$ is selfadjoint then ipso facto $p_{k}= \pm p_{n-k} \forall k$, which is practically the same as (1.4). We shall disprove this naive argument in $\S 3$ by means of counterexamples: a selfadjoint operator may very well have infinite nonselfadjoint factorizations (at least for $n \geq$ 3) and also have infinite selfadjoint factorizations "essentially" different from one another (for each $n \geq 2$ ).

In this note we present a proof of Assertion A which is directly inferred from recent results both by Trench [14] and the author [9] on canonical factorizations of disconjugate differential operators. In such a way we shall also obtain some information on the behavior of the coefficients $p_{k}$ at the endpoints of the interval which may prove useful in applications.

2. A rigorous proof of Assertion A. In addition to what has been pointed out in the introduction, certain nontrivial difficulties connected with the regularity of the coefficients also arise. As we consider the problem of selfadjointness in the classical framework we make the further assumption in (1.1) that

$$
a_{k} \in C^{k}(a, b) \quad(k=0, \ldots, n) .
$$

In this case the adjoint of $L$ is uniquely determined by the operator

$$
\begin{aligned}
L^{*} v \equiv & (-1)^{n}\left(a_{n}(t) v\right)^{(n)} \\
& +(-1)^{n-1}\left(a_{n-1}(t) v\right)^{(n-1)}+\cdots+a_{0}(t) v \quad \forall v \in C^{n}(a, b) .
\end{aligned}
$$


A crucial step in our proof is that (1.3) can be inferred from (1.2) which is obviously permissible only when the coefficients $p_{k}$ in (1.2) are sufficiently smooth, namely when

$$
p_{k} \in C^{\max (k, n-k)}(a, b) \quad(k=0, \ldots, n) .
$$

As a matter of fact the assumption made at the outset of the paper, $a_{k} \in$ $C^{0}(a, b) \forall k$, implies that $L$ has a factorization (1.2) where

$$
p_{k} \in C^{n-k}(a, b) \quad(k=0, \ldots, n)
$$

(see the standard factorization obtained by the use of Wronskians in [12]); but it is not at all obvious that (2.1) implies (2.3). For convenience we shall strengthen condition (2.1) as follows:

$$
a_{k} \in C^{n}(a, b) \quad(k=0, \ldots, n),
$$

since (2.5) easily implies (2.3). In fact, given any factorization of type (1.2), we select the special fundamental system of solutions of $L u=0$ represented by

$$
\begin{gathered}
u_{0}=1 / p_{0} ; \\
u_{k+1}(t)=\frac{1}{p_{0}(t)} \int_{T}^{t} \frac{d t_{1}}{p_{1}\left(t_{1}\right)} \ldots \int_{T}^{t_{k-1}} \frac{d t_{k}}{p_{k}\left(t_{k}\right)} \quad(k=1, \ldots, n-1),
\end{gathered}
$$

with $T$ arbitrarily chosen in $(a, b)$. Since (2.5) implies that all the solutions of $L u=0$ are of class $C^{2 n}(a, b)$, a simple induction argument on the functions $u_{k}$ shows that $p_{k} \in C^{2 n-k}(a, b), k=0, \ldots, n$; and this implies (2.3).

In our opinion, conditions (2.5) are far from necessary in establishing our main result which should remain valid for the standard hypothesis (2.1). However, a rigorous proof of this rests on the fact that (2.1) implies (2.3) in every factorization of $L$ : this is left an open question.

The factorizations considered in the sequel are of the form (1.2) and when they are said to be valid on an interval $(c, d)$ it is understood that the $p_{k}$ 's are strictly positive on $(c, d)$. Here is the main result:

THEOREM A. Let $L$ be a linear ordinary differential operator represented by (1.1), of order $n \geq 2$ and with real-valued coefficients $a_{k} \in C^{n}(a, b), k=0, \ldots, n$, where $(a, b)$ denotes an arbitrary (possibly unbounded) open interval and $a_{n} \neq 0$ on $(a, b)$. If $L$ is disconjugate and formally selfadjoint on $(a, b)$ the following are true:

(I) $L$ admits on $(a, b)$ of a selfadjoint factorization of the type (1.2) such that

$$
\int_{a}\left(1 / p_{k}\right)=+\infty \quad(k=1, \ldots, n-1)
$$

and of another selfadjoint factorization such that

$$
\int^{b}\left(1 / p_{k}\right)=+\infty \quad(k=1, \ldots, n-1) .
$$

Moreover any factorization of $L$ subject to conditions (2.6) or (2.7) is automatically selfadjoint. that

(II) On each interval $(a, b-\epsilon), \epsilon>0, L$ admits of a selfadjoint factorization such

$$
\int_{a}\left(1 / p_{k}\right)<+\infty \quad(k=1, \ldots, n-1)
$$


Similarly, on each interval $(a+\epsilon, b), \epsilon>0, L$ admits of a selfadjoint factorization such that

$$
\int^{b}\left(1 / p_{k}\right)<+\infty \quad(k=1, \ldots, n-1) .
$$

(III) When it is furthermore assumed that $L$ is disconjugate on $[a, b]$, see $[9$, Definition $3.5, p .164]$, then $L$ has a selfadjoint factorization on the whole interval $(a, b)$ satisfying $(2.8)$ and another such factorization satisfying (2.9).

NoTE. If $b=+\infty$, the symbol $(a, b-\epsilon)$ stands for an interval of the type $(a, \bar{b})$, $a<\bar{b}<+\infty$; analogous convention for $a=-\infty$.

ProOF. As in [9] we shall use the following locutions: factorization (1.2) is termed a canonical factorization of type (I) [resp. of type (II)] at the endpoint $a$ if the functions $p_{k}$ satisfy (2.6) [resp. (2.8)]. The analogous definitions at the endpoint $b$ are obtained by replacing (2.6) with (2.7) and (2.8) with (2.9). Now from [14, Theorem 1] it follows that $L$ has a canonical factorization of type (I) at $a$, which we shall again call (1.2). Since from our preliminary remarks all the $p_{k}$ 's are of class $C^{n}(a, b)$, we can state (see [1, p. 104]) that the formal adjoint of $L$ has a factorization (1.3) which is obviously canonical of type (I) at $a$. Using the assumption that $L$ is formally selfadjoint on $(a, b)$ we conclude that $L$ has the two factorizations on $(a, b)$, (1.2) and (1.3), both of type (I) at $a$. From the essential uniqueness of factorizations of type (I), [14, Theorem 1], it follows that the $p_{k}$ 's must satisfy (1.4). The same reasoning holds at the endpoint $b$ and also the last assertion in part $I$ is proved. We shall now proceed to part III. As far as factorizations of type (II) valid on $(a, b)$ are concerned, we cannot assert that any such factorization (if it exists at all) is selfadjoint (see part B of $\S 3$ ). But if $L$ is supposed disconjugate not only on $(a, b)$ but also on $[a, b]$, in the appropriate generalized sense, (note that one or both the endpoints $a$ and $b$ may be infinite or that not all the coefficients $a_{k}$ may be extended with continuity to the closure of the interval) then Theorems 3.1 and 3.2 in [9] imply that any canonical factorization of type (I) at $a$ [resp. at $b]$ is of type (II) at $b$ [resp. at $a$. But we have just proved that any factorization of type (I)-and there is always one such factorization [14]-is selfadjoint.

For the proof of part II we note that any operator disconjugate on $(a, b)$ is disconjugate in the generalized sense on $[a, b)$ and on $(a, b]$ (Levin [11, Lemma 2.3, p. 61]), hence, it is obviously disconjugate on any subinterval $[a, b-\epsilon]$ and $[a+\epsilon, b]$. Now part II immediately follows from part III.

Notice that in Theorem A there is no claim that all selfadjoint factorizations are canonical; counterexamples are given in part B of $\S 3$.

In Theorem $\mathrm{A}$ it is supposed $n \geq 2$; for $n=1$ it is trivial to directly verify that any operator $L$ of the form $L u \equiv a_{1}(t) u^{\prime}+a_{0}(t) u$, where $a_{0} \in C^{0}(a, b), a_{1} \in C^{1}(a, b)$, $a_{1} \neq 0$ on $(a, b)$, has only one (constant factors apart) factorization of the type $L u \equiv p_{1}\left(p_{0} u\right)^{\prime}$ and that $L$ is formally selfadjoint iff $p_{1}=p_{0}$. For $n=1$ the concept of canonical factorization is meaningless.

A possible application of Theorem $\mathrm{A}$ is that if $L$ is a disconjugate selfadjoint operator then all the special asymptotic results pertaining to the perturbed equation $L u=f(t, u)$ in $[\mathbf{7}, 8]$ are valid.

3. Counterexamples and complements. In this section all the above-mentioned counterexamples are given pointing out the difference between the cases $n=2$ and $n>2$. 
A. The $n=2$ case. It is well known that the second-order differential operator

$$
L u \equiv a_{2}(t) u^{\prime \prime}+a_{1}(t) u^{\prime}+a_{0}(t) u \quad \forall u \in C^{2}(a, b)
$$

where $a_{k} \in C^{k}(a, b), k=0,1,2$, is selfadjoint iff $a_{1}=a_{2}^{\prime}$. We can furthermore assert

THEOREM 3.1. Every operator $L$ of the type (3.1), selfadjoint and disconjugate on an open interval $(a, b)$, has only selfadjoint factorizations, which in turn are of the form (constant factors apart) $L u \equiv p\left[a_{2}(p u)^{\prime} / p^{2}\right]^{\prime}$ for some suitable function $p>0$ on $(a, b)$.

This can be proved in an elementary manner by expanding any factorization of $L, L u \equiv p_{2}\left[p_{1}\left(p_{0} u\right)^{\prime}\right]^{\prime}$, and comparing the so-obtained coefficients with those of the expression (3.1). The factorization

$$
u^{\prime \prime}(t) \equiv(t-c)^{-1}\left[(t-c)^{2}(u(t) /(t-c))^{\prime}\right]^{\prime}
$$

which holds on an interval $(a, b),-\infty<a<b \leq+\infty$, whatever constant $c<a$ has been chosen, actually shows the existence of infinitely-many selfadjoint factorizations essentially different one from the other.

B. The $n \geq 3$ case.

THEOREM 3.2. For each integer $n \geq 3$ and for each open interval $(a, b)$ there exists an nth-order constant-coefficient linear differential operator $L$ such that

(i) $L$ is disconjugate and selfadjoint on $(a, b)$;

(ii) $L$ has nonselfadjoint factorizations (possibly infinitely-many).

Proof. First case: $(a, b) \neq \mathbf{R}$. We may choose $L=d^{n} / d t^{n}$. Indeed if, for instance, $a>-\infty$ and $b \leq+\infty$ then $L$ admits on $(a, b)$ of the factorization

$$
L u \equiv(t-c)^{k-n}\left[(t-c)^{n}\left(\frac{u}{(t-c)^{k}}\right)^{(n-k)}\right]^{(k)}
$$

where $c$ is an arbitrarily chosen constant $\leq a$ and $k$ an integer, $0<k<n-1$. Factorization (3.2) is selfadjoint iff $k=n / 2$. We point out in passing that if $k=$ $n / 2(n$ even $\leq 4)$ then factorization (3.2) is selfadjoint on the interval $(c,+\infty)$ but it is not canonical at either $c$ or $+\infty$. On the other hand if $k \neq n / 2$ and if $c<a$ then it is canonical on $(a, b)$ of type (II) at $a$ (if $b<+\infty$ it is also canonical of type (II) at $b$ ) but not selfadjoint.

Second case: $(a, b)=\mathbf{R}$. We may choose as $L$ any operator with constant real coefficients such that, when $r_{1}, \ldots, r_{n}$ are the $n$ roots of its characteristic equation, each counted according to its multiplicity, the following properties hold:

(1) all the roots are real (disconjugacy condition);

(2) if $r$ is a root with multiplicity $\nu$ then $(-r)$ is also a root with the same multiplicity (selfadjointness condition);

(3) at least two roots are distinct.

In such a case it is easy to convince oneself that there exists a permutation of the $n$ roots, again called $r_{1}, \ldots, r_{n}$, such that $r_{k}-r_{k+1} \neq r_{n-k}-r_{n-k+1}$ for at least one integer $k \in\{1, \ldots, n-1\}$. Then the factorization given in Theorem 4.1 of [9] is not selfadjoint.

Note that in the second case the choice of an operator other than $d^{n} / d t^{n}$ is not an unnecessary complication but agrees with the conjecture formulated in [9, Remark 3 following Theorem 3.3, p. 165]. 
As instances of operators satisfying (1), (2), (3), we have $L=\left(D^{2}-1\right)^{n}$ or $L=$ $D\left(D^{2}-1\right)^{n}$, where $D=d / d t$.

C. Factorizations on compact intervals. Suppose $L$ to be defined by (1.1) with $a_{k} \in C^{n}[a, b],-\infty<a<b<+\infty$, and let $L$ be disconjugate and formally selfadjoint on $(a, b)$. What then can be said about factorizations of $L$ on $[a, b]$, i.e. with coefficients $p_{k}$ satisfying (1.4) on $[a, b]$ ? It is by no means true that if a factorization of $L$ holds [and in particular if it is selfadjoint] on $(a, b)$ then it holds automatically [and is selfadjoint] on $[a, b]$; in fact the essence of the problem is that the very continuity of the $p_{k}$ 's at the endpoints is dubious: glance for instance at (3.2).

In this direction we can give the following result when $L$ is assumed to be disconjugate also on $[a, b]$.

THEOREM 3.3. Let $L$ be the operator (1.1) with $a_{k} \in C^{0}[a, b](-\infty<a<b<$ $+\infty ; k=0, \ldots, n)$ and $a_{n} \neq 0$ on $[a, b]$, and let $L$ be disconjugate on $[a, b]$. Then $L$ has a factorization (1.2) where

$$
p_{k}>0 \text { on }[a, b] \text { and } p_{k} \in C^{n-k}[a, b] \text { for each } k=0, \ldots, n \text {. }
$$

Under the further assumption that $L$ is formally selfadjoint on $(a, b)$ and that $a_{k} \in$ $C^{n}[a, b] \forall k$, then $L$ has a selfadjoint factorization (1.2) on $[a, b]$ where the $p_{k}$ 's satisfy conditions (3.3), hence obviously

$$
\int_{a}^{b}\left(1 / p_{k}\right)<+\infty \quad \forall k .
$$

PROOF. Let $\bar{a}_{k}$ be an arbitrary extension of $a_{k}$ on $\mathbf{R}, \bar{a}_{k} \in C^{0}(\mathbf{R}), k=0, \ldots, n$, and let $\bar{L}$ be the operator

$$
\bar{L} u \equiv \bar{a}_{n}(t) u^{(n)}+\cdots+\bar{a}_{0}(t) u \quad \forall u \in C^{n}(\mathbf{R}) .
$$

By our assumptions $\bar{L}$ is disconjugate on $[a, b]$; hence, it follows from Lemma 7 in $[1$, p. 93] that there exists an $\epsilon>0$ such that $\bar{L}$ is disconjugate on $(a-\epsilon, b+\epsilon)$; this in turn, by Polya's characterization of disconjugacy, implies that $\bar{L}$ has a factorization of type (1.2) on $(a-\epsilon, b+\epsilon)$. Such a factorization is obviously also a factorization of the operator $L$ on $[a, b]$ satisfying (3.3)-for the smoothness of the $p_{k}$ 's see (2.4). $\bar{L}$.

The second part of the theorem follows by applying Theorem A to the operator

It is instructive to note that under the hypotheses of the theorem, $L$ cannot have a canonical factorization of type (I) at an endpoint, say $a$, with all the coefficients $p_{k} \in C^{0}[a, b)$. In fact, in this case the two conditions

$$
\begin{cases}p_{k} \in C^{0}[a, b) & (k=0, \ldots, n), \\ \int_{a}\left(1 / p_{k}\right)=+\infty & (k=1, \ldots, n-1)\end{cases}
$$

would imply $p_{k}(0)=0(k=1, \ldots, n-1)$; further, from

$$
L u=p_{n}\left[p_{n-1}\left(\cdots\left(p_{0} u\right)^{\prime} \cdots\right)^{\prime}\right]^{\prime} \equiv a_{n}(t) u^{(n)}+\cdots+a_{0}(t) u
$$

it follows that $a_{n}=p_{0} p_{1} \cdots p_{n}$, hence $a_{n}(0)=0$, contradicting one of the hypotheses. The conclusive step in the foregoing reasoning uses vitally the continuity of all the $p_{k}$ 's on $[a, b)$, otherwise we may exhibit as a counterexample the following 
factorization of the operator $L=d^{n} / d t^{n}$ :

$$
u^{(n)}(t) \equiv \frac{1}{t^{n-1}}\left[t^{2}\left(\ldots\left[t^{2}\left(\frac{u}{t^{n-1}}\right)^{\prime}\right]^{\prime} \ldots\right)^{\prime}\right]^{\prime}
$$

which has the following peculiarities:

(i) the operator $L u \equiv u^{(n)}$ has coefficients $a_{k} \in C^{\infty}(\mathbf{R}), a_{k} \neq 0$ on $\mathbf{R}(k=0, \ldots, n)$;

(ii) the coefficients $p_{k}$ satisfy

$$
\begin{cases}p_{k} \in C^{\infty}[0,+\infty) & (k=1, \ldots, n-1), \\ p_{k}(0)=0 & (k=1, \ldots, n-1)\end{cases}
$$

(hence $\left.\int_{0}\left(1 / p_{k}\right)=+\infty, k=1, \ldots, n-1\right)$.

Note the difference between (3.4) and (3.5).

ACKNOWLEDGEMENT. The author thanks the referee for his helpful suggestions.

\section{REFERENCES}

1. W. A. Coppel, Disconjugacy, Lecture Notes in Math., vol. 220, Springer-Verlag, Berlin, 1971.

2. G. Darboux, Leçons sur la théorie générale des surfaces, 2me partie, 2me edition, GauthierVillars, Paris, 1915.

3. G. Frobenius, Ueber den Begriff der Irreductibilität in der Theorie der linearen Differentialgleichungen, J. für Math. 76 (1873), 236-270.

4. __ Ueber die Determinante mehrerer Funktionen einer Variabeln, J. für Math. 77 (1874), 245-257.

5. $\ldots$, Ueber die regulären Integrale der linearen Differentialgleichungen, J. für Math. 80 (1875), 317-333.

6. ___ Ueber adjungirte lineare Differentialausdrücke, J. für Math. 85 (1878), 185-213.

7. A. Granata, Singular Cauchy problems and asymptotic behaviour for a class of $n$-th order differential equations, Funkcial. Ekvac. 20 (1977), 193-212.

8. - Corrigendum and addendum: Singular Cauchy problems and asymptotic behaviour for a class of the $n$-th order differential equations, Funkcial. Ekvac. 22 (1979), 351-354.

9. _ Canonical factorizations of disconjugate differential operators, SIAM J. Math. Anal. 11 (1980), 160-172.

10. E. L. Ince, Ordinary differential equations, Dover, New York, 1956.

11. A. Yu. Levin, Non-oscillation of solutions of the equation $x^{(n)}+p_{1}(t) x^{(n-1)}+\cdots+p_{n}(t) x=0$, Uspehi Mat. Nauk 24 (1969), 43-96; Russian Math. Surveys 24 (1969), 43-99.

12. G. Polya, On the mean-value theorem corresponding to a given linear homogeneous differential equation, Trans. Amer. Math. Soc. 24 (1922), 312-324.

13. L. Schlesinger, Handbuch der Theorie der linearen Differentialgleichungen, Band I, Teubner, Leipzig, 1895.

14. W. F. Trench, Canonical forms and principal systems for general disconjugate equations, Trans. Amer. Math. Soc. 189 (1974), 319-327.

Dipartimento di MAtematica, Università della Calabria, 87036 ARCAVACATA DI RENDE (COSENZA), ITALY 